\title{
Single soft gluon emission at two loops
}

\section{Ye Li and Hua Xing Zhu}

SLAC National Accelerator Laboratory, Stanford University, Stanford, CA 94309, U.S.A.

E-mail: yli@slac.stanford.edu, hxzhu@slac.stanford.edu

ABSTRACT: We study the single soft-gluon current at two loops with two energetic partons in massless perturbative QCD, which describes, for example, the soft limit of the twoloop amplitude for $g g \rightarrow H g$. The results are presented as Laurent expansions in $\epsilon$ in $D=4-2 \epsilon$ spacetime dimension. We calculate the expansion to order $\epsilon^{2}$ analytically, which is a necessary ingredient for Higgs production at hadron colliders at next-to-nextto-next-to-leading order in the soft-virtual approximation. We also give two-loop results of the single soft-gluon current in $\mathcal{N}=4$ Super-Yang-Mills theory, and find that it has uniform transcendentality. By iteration relation of splitting amplitudes, our calculations can determine the three-loop single soft-gluon current to order $\epsilon^{0}$ in $\mathcal{N}=4$ Super-YangMills theory in the limit of large $N_{c}$.

KeYwords: QCD, Parton Model

ArXiv ePrint: 1309.4391 


\section{Contents}

1 Introduction $\quad 1$

2 Review of the soft-gluon current $\quad 2$

3 Calculation of the two-loop soft-gluon current 3

3.1 Warm up: one-loop soft-gluon current 4

3.2 Two-loop soft-gluon current 5

3.3 Single soft-gluon current in $\mathcal{N}=4$ super-Yang-Mills theory 8

4 Conclusion $\quad 10$

$\begin{array}{ll}\text { A Evaluation of the master integral } I_{3} \text { to order } \epsilon^{2} & 11\end{array}$

\section{Introduction}

Amplitudes in gauge theory develop infrared divergences when one or multiple external partons become soft/collinear. Fortunately, in the soft/collinear limit, there exist universal factorization properties for such amplitudes, which are the foundation of higher-order perturbative-QCD computations. Extensive discussion on the factorization of gauge-theory amplitudes in the infrared region can be found, for example, in refs. [1-24].

In QCD, the radiation of an arbitrary number of soft gluons off a tree-level amplitude can be obtained using the well-known Berends-Giele recursion relation [3]. Due to the long-range properties of soft gluon radiation, amplitudes in the soft limit have non-local color correlations. Compact expressions for tree-level amplitudes with two soft partons have been obtained in the color space formalism in ref. [7]. Emission of a single soft gluon from a generic one-loop amplitude have also been studied by several groups [8, 12, 14, 15]. These results have been proven to be important in the program of next-to-next-to-leading order (NNLO) QCD computations for jet physics, see for example refs. [25-31].

While the NNLO revolution is under way, there is strong motivation for going one order in $\alpha_{s}$ further. This is driven by both experimental and theoretical demands. On the experimental side, the discovery of Higgs boson marks one of the most important progress in particle physics in the last few decades [32, 33]. It's certainly important to give the most precise theoretical prediction for its production cross section. On the theory side, uncertainties estimated by scale variation for Higgs production is around $\pm 10 \%$ at NNLO [34-37], and improved to $\pm 7 \%$ by including soft-gluon resummation up to nextto-next-to-leading logarithmic accuracy [38]. Further decreasing the scale uncertainties to percent level requires the computation of next-to-next-to-next-to-leading order (NNNLO) QCD corrections. 
In this paper we consider single soft-gluon radiation at two loops, which plays an important role in NNNLO QCD corrections, similar to the one-loop soft-gluon current does in NNLO computations, see for example, refs. [25-27, 39]. To simplify the situation, we confine ourselves to the case that only two hard partons are present. This corresponds to the cases such as $e^{+} e^{-} \rightarrow$ dijet, deep-inelastic scattering, or Drell-Yan/Higgs production at hadron collider. Previously, such amplitudes have been derived [19] by taking the soft limit of collinear splitting amplitudes at two loops to order $\epsilon^{0}$, using the two-loop aamplitudes for $\gamma^{*} \rightarrow q \bar{q} g[40,41]$ and $H \rightarrow g g g$ [42]. However, for a NNNLO computation, one needs the Laurent expansion in $\epsilon$ through the $\epsilon^{2}$ terms, which we have given for the first time in this paper. Our results for the two-loop soft amplitude agree with the soft limit of the two-loop splitting amplitudes $[18,19]$ through the $\epsilon^{0}$ terms, serving as a strong check of our calculation.

As a by-product, we obtain the soft-gluon current in $\mathcal{N}=4$ Super-Yang-Mills theory to order $\epsilon^{2}$, which coincides with the QCD result at leading transcendentality. We also derive the soft limit of splitting amplitudes at three loops through order $\epsilon^{0}$ at leading order of $N_{c} \rightarrow \infty$, using the results of refs. [43, 44].

The paper is organized as follows. In section 2, we review the general result on the factorization of the single soft-gluon current at tree level and one loop. In section 3 we calculate the soft-gluon current to two loops. We conclude at section 4 . We present some details for the computation of one of the master integral in the appendix.

\section{Review of the soft-gluon current}

In this section we review the factorization of amplitudes in the soft limit, closely following the notation in ref. [15]. It's well-known $[2,4]$ that tree-level QCD amplitudes with two hard partons and one soft gluon can be written as

$$
\left|\mathcal{M}^{(0)}\left(q, p_{1}, p_{2}\right)\right|^{2} \simeq 4 g_{s}^{2} \mu^{2 \epsilon} C_{R} S_{12}^{(0)}(q)\left|\mathcal{M}^{(0)}\left(p_{1}, p_{2}\right)\right|^{2}
$$

where $S_{12}^{(0)}(q)=\frac{p_{1} \cdot p_{2}}{2\left(q \cdot p_{1}\right)\left(q \cdot p_{2}\right)}$, and $\mathcal{M}^{(0)}\left(q, p_{1}, p_{2}\right)$ is the tree-level amplitude for 2 hard partons (massless quark or gluon) and one soft gluon, and $\mathcal{M}^{(0)}\left(p_{1}, p_{2}\right)$ is the corresponding amplitude with the soft gluon stripped off. Dependence of the amplitudes on the extra colorless particles in the process is left implicit. The symbol $\simeq$ means that we have neglected terms that are less singular than $1 / q^{2} \cdot g_{s}$ is the strong coupling constant, $\mu$ is the mass scale introduced by continuing the space-time dimension to $D=4-2 \epsilon$ dimension. $C_{R}$ is the quadratic Casimir invariant. $C_{R}=C_{A}$ if parton 1 is a gluon, $C_{R}=C_{F}$ if parton 1 is a quark, where $C_{A}=N_{c}$ and $C_{F}=\frac{N_{c}^{2}-1}{2 N_{c}}$, with $N_{c}$ being the number of color. Note that the functional dependence of the eikonal function $S_{12}^{(0)}(q)$ is uniquely determined by its invariance under the rescaling of $p_{1}$ and $p_{2}$, which is a simple consquence of the QCD Feynman rule in the eikonal limit. In our convention, all momenta are massless and have positive-definite energies. The generalization of eq. (2.1) to processes with any number of hard partons can be found, for example, in ref. [7]. 
At the one-loop level, eq. (2.1) receives quantum corrections, which can be written as

$$
\begin{aligned}
& \mathcal{M}^{(0)}\left(q, p_{1}, p_{2}\right) \mathcal{M}^{(1)}\left(q, p_{1}, p_{2}\right)^{*}+\text { c.c. } \\
& \simeq\left(4\left(g_{s} \mu^{\epsilon}\right)^{2} C_{R} S_{12}^{(0)}(q) \mathcal{M}^{(0)}\left(p_{1}, p_{2}\right) \mathcal{M}^{(1)}\left(p_{1}, p_{2}\right)^{*}+\text { c.c. }\right) \\
& \quad+\left(4\left(g_{s} \mu^{\epsilon}\right)^{2} C_{R} S_{12}^{(1)}(q)\left|\mathcal{M}^{(0)}\left(p_{1}, p_{2}\right)\right|^{2}+\text { c.c. }\right),
\end{aligned}
$$

where c.c. denotes complex conjugate. $\mathcal{M}^{(i)}$ is the $i$ th order in $\alpha_{s}$ unrenormalized amplitudes in dimensional regularization, where UV and IR divergences are simutaneously regularized by the dimensional regularization parameter $\epsilon$. The one-loop corrections to the eikonal function have been calculated to be $[8,12,14,15]$

$$
S_{12}^{(1)}(q)=-S_{12}^{(0)}(q) \frac{\alpha_{s}}{4 \pi} C_{A} S_{\epsilon} \frac{e^{\epsilon \gamma_{E}} \Gamma^{3}(1-\epsilon) \Gamma^{2}(1+\epsilon)}{\epsilon^{2} \Gamma(1-2 \epsilon)},
$$

where

$$
S_{\epsilon}=\left(4 \pi e^{-\gamma_{E}} e^{i \sigma_{12} \pi} \mu^{2} S_{12}^{(0)}(q)\right)^{\epsilon},
$$

and $\sigma_{12}=-1$ if both $p_{1}$ and $p_{2}$ are incoming, otherwise $\sigma_{12}=1$. Note that the oneloop eikonal function doesn't depend on $C_{R}$, which may be explained by the non-abelian exponentiation theorem $[45,46]$, if one replaces the polarization summation for the soft gluon by a cut propagator. Eq. (2.3), and its generalization to processes with any number of hard partons have been used, for example, in the calculation of soft-virtual approximation to Higgs production at NNLO [47-49], in the calculation of the two-loop soft function in soft-collinear-effective theory [50-53], and in the construction of subtraction term in general NNLO corrections [25-27, 39].

\section{Calculation of the two-loop soft-gluon current}

At two loops, the factorized soft-gluon current has the form

$$
\begin{aligned}
\mathcal{M}^{(0)}\left(q, p_{1}, p_{2}\right) \mathcal{M}^{(2)}\left(q, p_{1}, p_{2}\right)^{*} & + \text { c.c. } \\
\simeq 4\left(g_{s} \mu^{\epsilon}\right)^{2} C_{R}\left[\left(S_{12}^{(0)}\right.\right. & \left.(q) \mathcal{M}^{(0)}\left(p_{1}, p_{2}\right) \mathcal{M}^{(2)}\left(p_{1}, p_{2}\right)^{*}+\text { c.c. }\right) \\
& +\left(S_{12}^{(1)}(q) \mathcal{M}^{(0)}\left(p_{1}, p_{2}\right) \mathcal{M}^{(1)}\left(p_{1}, p_{2}\right)^{*}+\text { c.c. }\right) \\
& \left.+\left(S_{12}^{(2)}(q)\left|\mathcal{M}^{(0)}\left(p_{1}, p_{2}\right)\right|^{2}+\text { c.c. }\right)\right] .
\end{aligned}
$$

The two-loop generalization is consistent with the soft limit of two-loop collinear splitting amplitudes $[18,19]$. For the latter, it has been shown that similar factorization form holds to all orders in $\alpha_{s}[9]$. The two-loop eikonal function, $S_{12}^{(2)}(q)$, is known through the order $\epsilon^{0}$ terms by taking the soft limit of the two-loop collinear splitting amplitudes $[18,19]$ or the two-loop squared amplitudes for $\gamma^{*} \rightarrow q \bar{q} g$ and $H \rightarrow g g g$ [40-42]. However, for computation accurate to NNNLO, one also needs the order $\epsilon$ and order $\epsilon^{2}$ terms. In this section, we calculate the Laurent expansions of $S_{12}^{(2)}(q)$ in $\epsilon$ through order $\epsilon^{2}$, using a method different from refs. [19]. 
In ref. [15], the one-loop soft-gluon current is derived by taking the eikonal approximation of the integrand of the amplitudes before the loop integrals are carried out. This has the advantage that the one-loop eikonal function can be directly obtained without the subtraction of the product of the tree-level eikonal function and the one-loop squared amplitude, that is, the second line of eq. (2.2). The same procedure can be used in the calculation of the two-loop eikonal function.

Specifically, we generate the integrand corresponding to the interference of tree-level and two-loop amplitudes, the first line of eq. (3.1). For this purpose, we consider the process $\gamma^{*} \rightarrow q\left(p_{1}\right) \bar{q}\left(p_{2}\right) g(q)$, keeping in mind that the eikonal function is independent of the colorless particles in the process. Summation of polarization for the external gluon is done in Feynman gauge. We then take the eikonal approximation of the integrand, assumming that the energy of the internal and external gluons are parametrically smaller then $p_{1}^{0}$ and $p_{2}^{0}$. The integrand after the eikonal approximation is taken can also be generated by treating $q\left(p_{1}\right)$ and $q\left(p_{2}\right)$ as two out-going Wilson lines, whose directions are given by $p_{1}^{\mu} / p_{1}^{0}$ and $p_{2}^{\mu} / p_{2}^{0}$. We have checked that this indeed gives the same integrand. ${ }^{1}$ We note that after the eikonal approximation is taken on the right hand side of eq. (3.1), the second and the third lines of it vanish. The reason is that $\mathcal{M}^{(1)}\left(p_{1}, p_{2}\right)$ and $\mathcal{M}^{(2)}\left(p_{1}, p_{2}\right)$ become scaleless integrals and vanish identically in dimensional regularization. Therefore, The two-loop eikonal function can be obtained by evaluating the resulting integrand, without the need of subtraction.

\subsection{Warm up: one-loop soft-gluon current}

For the convenience of reader, we reproduce the one-loop results in this section. At one loop, there is only one non-zero diagram (from now on eikonal approximation is assumed for the integrand), which is depicted in figure 1. All the remaining diagrams are zero in dimensional regularization, because their loop integrals are scaleless. One example of such a vanishing diagram is depicted in figure 2 . We note that the external soft-gluon momentum only enters the loop integral through $q \cdot p_{1}$. However, the invariance of the integral under the rescaling of $p_{1}$ and $p_{2}$ demands that a factor of $\left(\frac{\mu^{2}\left(p_{1} \cdot p_{2}\right)}{\left(q \cdot p_{1}\right)\left(q \cdot p_{2}\right)}\right)^{\epsilon}$ must be generated per loop. This is impossible for this diagram, leading to the conclusion that it must vanish.

We calculate the interference between the one-loop non-zero diagram, figure 1, and the tree-level diagrams in figure 3. The one-loop eikonal function can then be extracted from the one-loop integral in the interference term, which, after some simplification, reads

$$
S_{12}^{(1)}(q)=i 4 g_{s}^{2} C_{A}\left(p_{1} \cdot p_{2}\right) \mu^{2 \epsilon} \int \frac{d^{D} k}{(2 \pi)^{D}} \frac{1}{\left[2 k \cdot p_{1}\right]\left[2(q-k) \cdot p_{2}\right]\left[k^{2}\right]\left[(k-q)^{2}\right]},
$$

where the Feynman prescription $i 0^{+}$is implicitly understood for all propagators in square brackets, for example, $\left[k^{2}\right] \equiv k^{2}+i 0^{+}$. Carrying out the loop integral, we reproduce the one-loop eikonal function in eq. (2.3).

\footnotetext{
${ }^{1}$ We use QGRAF [54] extensively in generating various diagrams.
} 


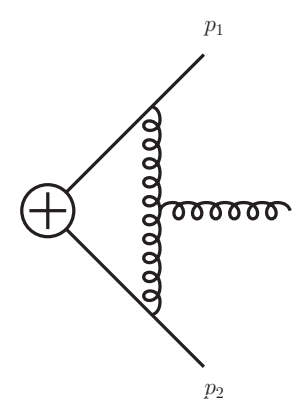

Figure 1. Non-vanishing diagram for soft gluon emission at one-loop. Solid line are quark/antiquark lines in the high energy limit.

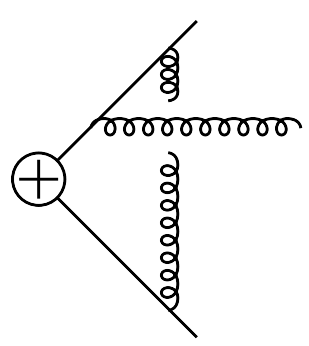

Figure 2. Diagram which vanishes in dimensional regularization.
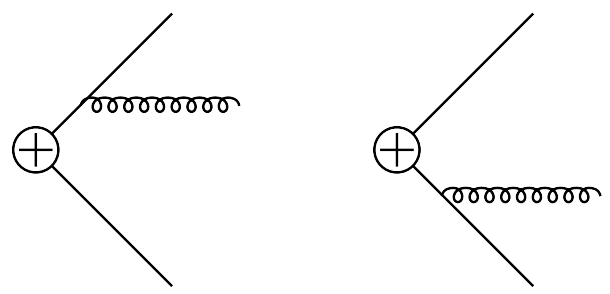

Figure 3. Tree-level diagrams for single soft gluon emission.

\subsection{Two-loop soft-gluon current}

As explained above, the two-loop eikonal function $S_{12}^{(2)}(q)$ can be extracted from the calculation of the non-vanishing diagrams at two-loop level, as depicted in figure 4. The grey blobs represent all possible two-point and three point insertions, where no eikonal approximation is made. We include $N_{f}$ flavour of massless fermions and $N_{s}$ flavour of massless scalar in the blob, besides the gluon. In QCD, $N_{f}=5, N_{s}=0$. Before describing the calculation of these diagrams, we comment on the diagrams that vanish identically. There are two classes of vanishing diagrams. The first class vanishes due to color or Lorentz algebra. An example of it is depicted in figure 5(a). The second class vanishes because the corresponding loop integral is scaleless, as in figure 5(b). Because of the vanishing of these two classes of diagrams, the actual number of diagrams that need to be evaluated is significantly reduced.

We now come to the actual evaluation of the non-zero diagrams in figure 4 . We calculate the interference terms between the tree-level diagrams in figure 3 and the twoloop diagrams in figure 4. After the evaluation of color factor and kinematical factor, 

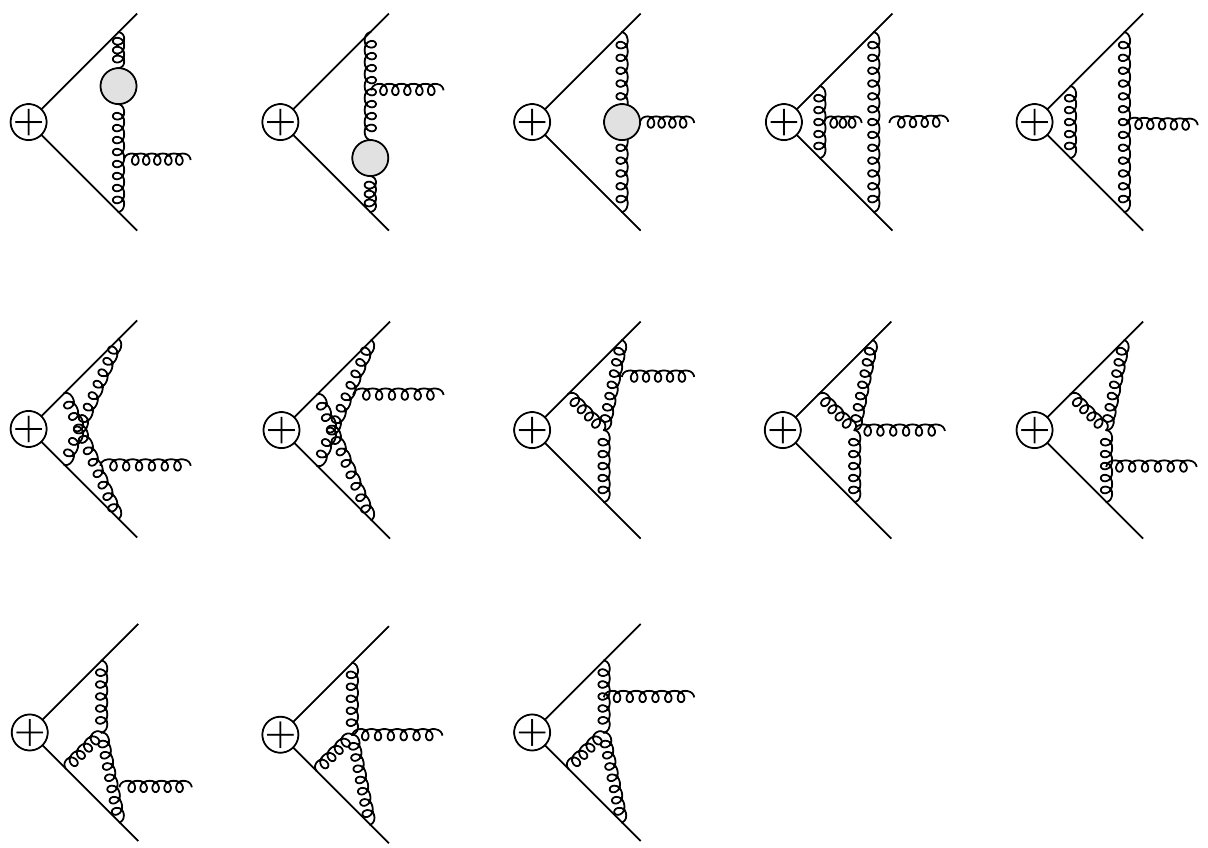

Figure 4. Two-loop non-vanishing diagrams for single soft gluon emission.
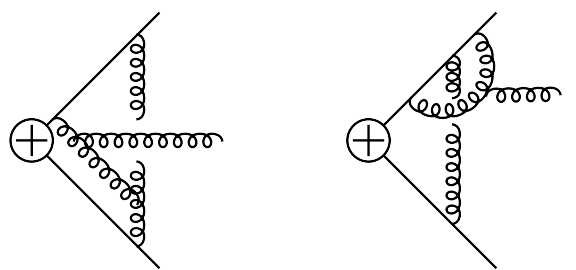

Figure 5. Examples of diagrams which vanish identically.

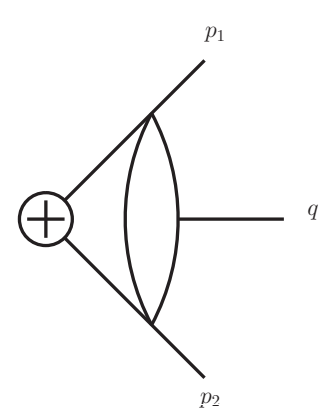

(a) $I_{1}$

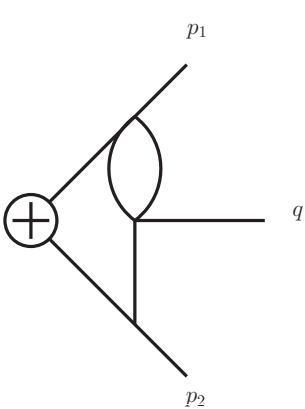

(b) $I_{2}$

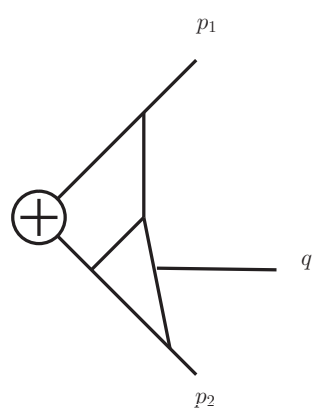

(c) $I_{3}$

Figure 6. Master integrals encountered in the computation. Eikonal approximations are taken on the directions $p_{1}$ and $p_{2}$.

the resulting loop integrals are reduced to three master integrals in figure 6 . To that end, we use the techniques of Integration-By-Parts (IBP) $[55,56]$, implemented in the MATHEMATICA package FIRE [57] using the Laporta algorithm [58]. The reduction to master integrals has also been cross checked using a different MATHEMATICA package 
LiteRed [59]. The results after the IBP reduction procedure can be written as

$$
\begin{aligned}
S_{12}^{(2)}(q)= & g_{s}^{4} \frac{p_{1} \cdot p_{2}}{\left(q \cdot p_{1}\right)\left(q \cdot p_{2}\right)} \times\left\{C _ { A } N _ { f } \left[\frac{2(-7+2 D)\left(12-6 D+D^{2}\right)}{(-6+D)(-3+D)(-2+D)(-1+D)} I_{1}\right.\right. \\
& \left.-\frac{6(-4+D)^{2}}{(-6+D)(-2+D)(-1+D)} I_{2}\right]+C_{A} N_{s}\left[-\frac{(-7+2 D)\left(-4-4 D+D^{2}\right)}{2(-6+D)(-2+D)(-1+D)} I_{1}\right. \\
& \left.+\frac{3(-4+D)^{2}}{(-6+D)(-2+D)(-1+D)} I_{2}\right]+C_{A}^{2}\left[+\frac{8}{3} I_{3}\right. \\
& \quad-\frac{\left(2(-156+D(72+D(11+(-9+D) D)))-3(-4+D)^{3} D_{s}\right)}{(-6+D)(-4+D)(-2+D)(-1+D)} I_{2} \\
& +\left(\frac{(-7+2 D)\left(504-1308 D+874 D^{2}-213 D^{3}+17 D^{4}\right)}{3(-6+D)(-4+D)(-3+D)(-2+D)(-1+D)}\right. \\
& \left.\left.\left.\quad-\frac{(-7+2 D)\left(-4-4 D+D^{2}\right) D_{s}}{2(-6+D)(-2+D)(-1+D)}\right) I_{1}\right]\right\}
\end{aligned}
$$

The parameter $D_{s}$ selects the particular variant of dimensional regularization. For $D_{s}=$ $4-2 \epsilon$ the scheme is the conventional dimensional regularization scheme, while for $D_{s}=4$ it is the four-dimensional helicity scheme (FDH) [60, 61].

There are three master integrals encountered in this computation. They are defined as

$$
\begin{aligned}
& I_{1}=\mu^{4 \epsilon} \int \frac{d^{D} k_{1} d^{D} k_{2}}{(2 \pi)^{2 D}} \frac{p_{1} \cdot p_{2}}{\left[2 k_{1} \cdot p_{1}\right]\left[2\left(q-k_{1}\right) \cdot p_{2}\right]\left[k_{2}^{2}\right]\left[\left(k_{2}-q\right)^{2}\right]\left[\left(k_{1}-k_{2}\right)^{2}\right]} \\
& I_{2}=\mu^{4 \epsilon} \int \frac{d^{D} k_{1} d^{D} k_{2}}{(2 \pi)^{2 D}} \frac{p_{1} \cdot p_{2}}{\left[2 k_{1} \cdot p_{1}\right]\left[2\left(q-k_{1}\right) \cdot p_{2}\right]\left[k_{1}^{2}\right]\left[k_{2}^{2}\right]\left[\left(k_{1}+k_{2}-q\right)^{2}\right]} \\
& I_{3}=\mu^{4 \epsilon} \int \frac{d^{D} k_{1} d^{D} k_{2}}{(2 \pi)^{2 D}} \frac{\left(q \cdot p_{1}\right)\left(q \cdot p_{2}\right)^{2}}{\left[2 k_{1} \cdot p_{1}\right]\left[2\left(q-k_{1}\right) \cdot p_{2}\right]\left[2\left(k_{2}+q\right) \cdot p_{2}\right]\left[k_{2}^{2}\right]\left[\left(k_{1}+k_{2}\right)^{2}\right]\left[\left(k_{2}+q\right)^{2}\right]}
\end{aligned}
$$

where $i 0^{+}$dependences in the propagators are understood. The first two masters are calculated to all orders in $\epsilon$. For the last master integral, we give the Laurent expansion of it to order $\epsilon^{2}$, which is the order relevant for NNNLO computation. The details of the computation of the last integral are presented in the appendix. Here we only list the results for the three master integrals:

$$
\begin{gathered}
I_{1}=-\frac{1}{\left(16 \pi^{2}\right)^{2}} S_{\epsilon}^{2} \frac{e^{2 \epsilon \gamma_{E}} \Gamma^{2}(1-2 \epsilon) \Gamma^{2}(1-\epsilon) \Gamma^{2}(1+2 \epsilon)}{8 \epsilon^{3}(1-4 \epsilon) \Gamma(1-4 \epsilon)} \\
I_{2}=-\frac{1}{\left(16 \pi^{2}\right)^{2}} S_{\epsilon}^{2} \frac{e^{2 \epsilon \gamma_{E}} \Gamma(1-2 \epsilon) \Gamma^{3}(1-\epsilon) \Gamma^{2}(1+2 \epsilon)}{8 \epsilon^{3}(1-2 \epsilon) \Gamma(1-3 \epsilon)} \\
I_{3}=-\frac{1}{\left(16 \pi^{2}\right)^{2}} S_{\epsilon}^{2}\left[-\frac{1}{8 \epsilon^{4}}-\frac{5 \zeta_{2}}{16 \epsilon^{2}}+\frac{25 \zeta_{3}}{48 \epsilon}-\frac{17 \zeta_{4}}{16}+\epsilon\left(\frac{67 \zeta_{2} \zeta_{3}}{48}+\frac{319 \zeta_{5}}{80}\right)\right. \\
\left.+\epsilon^{2}\left(\frac{101 \zeta_{3}^{2}}{36}+\frac{1723 \zeta_{6}}{256}\right)+\mathcal{O}\left(\epsilon^{3}\right)\right]
\end{gathered}
$$

where $\zeta_{s}$ is the Riemann zeta vaule, $\zeta_{s}=\sum_{n=1}^{\infty} \frac{1}{n^{s}}$. It's interesting to note that $I_{3}$ coincides with the soft limit of the corresponding master integral in full QCD, where no eikonal approximation is taken in the denominator. The latter was calculated in ref. [62] to order $\epsilon^{0}$. 
This is probably due to the fact that the divergences in $I_{3}$ have only infrared origin. While we have only presented the Laurent expansions of $I_{3}$ to order $\epsilon^{2}$ analytically, the higherorder terms can easily be obtained numerically, using its two-fold Mellin-Barnes integral representation derived in the appendix, and the MBintegrate routine of Czakon [63]. For example, the next three terms in the $\epsilon$ expansion of $I_{3}$ are given by

$$
(82.1443689 \pm 0.0000007) \epsilon^{3}+(198.904248 \pm 0.000002) \epsilon^{4}+(726.325910 \pm 0.000007) \epsilon^{5}
$$

However, it's difficult to convert them into Riemann zeta values due to lack of significant digits.

Substituting the master integral into eq. (3.3) and setting $N_{s}=0$, we obtain the twoloop eikonal function in QCD in the conventional dimensional regularization scheme $(D=$ $\left.D_{s}=4-2 \epsilon\right)$,

$$
\begin{aligned}
& S_{12}^{(2)}(q)=S_{12}^{(0)}(q)(\left.\frac{\alpha_{s}}{4 \pi}\right)^{2} S_{\epsilon}^{2}\left\{C _ { A } N _ { f } \left[\frac{1}{6 \epsilon^{3}}+\frac{5}{18 \epsilon^{2}}+\frac{19}{54 \epsilon}+\frac{\zeta_{2}}{6 \epsilon}+\frac{65}{162}+\frac{5 \zeta_{2}}{18}-\frac{31 \zeta_{3}}{9}\right.\right. \\
&+\epsilon\left(-\frac{35 \zeta_{2}}{54}-\frac{155 \zeta_{3}}{27}-\frac{185 \zeta_{4}}{24}+\frac{211}{486}\right) \\
&\left.+\epsilon^{2}\left(-\frac{31}{9} \zeta_{3} \zeta_{2}-\frac{367 \zeta_{2}}{162}-\frac{994 \zeta_{3}}{81}-\frac{925 \zeta_{4}}{72}-\frac{511 \zeta_{5}}{15}+\frac{665}{1458}\right)\right] \\
&+C_{A}^{2}\left[\frac{1}{2 \epsilon^{4}}-\frac{11}{12 \epsilon^{3}}+\frac{-\frac{67}{36}+\zeta_{2}}{\epsilon^{2}}+\frac{-\frac{193}{54}-\frac{11 \zeta_{2}}{12}-\frac{11 \zeta_{3}}{6}-\frac{571}{81}-\frac{67 \zeta_{2}}{36}+\frac{341 \zeta_{3}}{18}}{+\frac{7 \zeta_{4}}{8}+\epsilon\left(-\frac{7}{6} \zeta_{3} \zeta_{2}-\frac{139 \zeta_{2}}{54}+\frac{2077 \zeta_{3}}{54}+\frac{2035 \zeta_{4}}{48}-\frac{247 \zeta_{5}}{10}-\frac{3410}{243}\right)}\right. \\
&+\epsilon^{2}\left(-\frac{205 \zeta_{3}^{2}}{18}+\frac{341 \zeta_{2} \zeta_{3}}{18}+\frac{6388 \zeta_{3}}{81}-\frac{436 \zeta_{2}}{81}+\frac{12395 \zeta_{4}}{144}+\frac{5621 \zeta_{5}}{30}\right. \\
&\left.\left.\left.\quad-\frac{3307 \zeta_{6}}{48}-\frac{20428}{729}\right)\right]+\mathcal{O}\left(\epsilon^{3}\right)\right\} .
\end{aligned}
$$

Eq. (3.7) is the main result of this paper. We remind the reader that this result is for the unrenormalized amplitudes. To obtain the renormalized ones, one only needs to perform a renormalization on the strong coupling $\alpha_{s}$. We have checked eq. (3.7) against the two-loop splitting amplitudes in the soft limit calculated in refs. $[18,19]$, and found full agreement to order $\epsilon^{0}$. To the best of our knowledge, the order $\epsilon$ and $\epsilon^{2}$ terms presented in this paper are new.

\subsection{Single soft-gluon current in $\mathcal{N}=4$ super-Yang-Mills theory}

Using the generic results presented above, it's straightforward to obtain the single softgluon current in $\mathcal{N}=4$ Super-Yang-Mills theory, by setting $N_{f}=4 C_{A}, N_{s}=6 C_{A}$, and $D_{s}=4$ (corresponding to FDH scheme $[60,61]$ ) in eq. (3.3):

$$
S_{12, \mathcal{N}=4}^{(2)}(q)=g_{s}^{4} C_{A}^{2} \frac{p_{1} \cdot p_{2}}{\left(q \cdot p_{1}\right)\left(q \cdot p_{2}\right)}\left[-\frac{1-4 \epsilon}{3 \epsilon} I_{1}+\frac{1-2 \epsilon}{\epsilon} I_{2}+\frac{8}{3} I_{3}\right] .
$$


This result is remarkably simple. It becomes obvious that the result in $\mathcal{N}=4$ Super-YangMills theory has uniform transcendentality, as long as $I_{3}$ does. Substituting the explicit form of the master integrals into eq. (3.8), we obtain

$$
\begin{aligned}
S_{12, \mathcal{N}=4}^{(2)}(q)= & S_{12}^{(0)}(q)\left(\frac{\alpha_{s}}{4 \pi}\right)^{2} S_{\epsilon}^{2} C_{A}^{2} \\
\times\left[\frac{1}{2 \epsilon^{4}}+\right. & \frac{\zeta_{2}}{\epsilon^{2}}-\frac{11 \zeta_{3}}{6 \epsilon}+\frac{7 \zeta_{4}}{8}+\epsilon\left(-\frac{7 \zeta_{2} \zeta_{3}}{6}-\frac{247 \zeta_{5}}{10}\right) \\
& \left.+\epsilon^{2}\left(-\frac{205 \zeta_{3}^{2}}{18}-\frac{3307 \zeta_{6}}{48}\right)+\mathcal{O}\left(\epsilon^{3}\right)\right] .
\end{aligned}
$$

We note that at leading transcendentality, the eikonal soft function in $\mathcal{N}=4$ Super-YangMills theory coincides with the one in QCD through $\epsilon^{2}$, as also happens in some other context [64].

It's also interesting to notice that eq. (3.9) can be reorganized as $[65]^{2}$

$$
\begin{aligned}
S_{12, \mathcal{N}=4}^{(2)}(q) & \equiv 4 S_{12}^{(0)}(q)\left(\frac{\alpha_{s}}{4 \pi}\right)^{2} S_{\epsilon}^{2} C_{A}^{2} r_{S}^{(2)}(\epsilon) \\
& =4 S_{12}^{(0)}(q)\left(\frac{\alpha_{s}}{4 \pi}\right)^{2} S_{\epsilon}^{2} C_{A}^{2}\left(\frac{1}{2}\left(r_{S}^{(1)}(\epsilon)\right)^{2}+f(\epsilon) r_{S}^{(1)}(2 \epsilon)\right)+\mathcal{O}(\epsilon),
\end{aligned}
$$

where $r_{S}^{(1)}(\epsilon)=-e^{\epsilon \gamma_{E}} \frac{\Gamma^{3}(1-\epsilon) \Gamma^{2}(1+\epsilon)}{2 \epsilon^{2} \Gamma(1-2 \epsilon)}$ is the soft limit of the one-loop collinear splitting amplitudes in $\mathcal{N}=4$ Super-Yang-Mills theory (up to an overall $z$-dependent factor, same below), and $f(\epsilon)=-\sum_{i=1}^{\infty} \zeta_{i+1} \epsilon^{i-1}$ [65]. Eq. (3.10) makes explicit the iterative structure of $\mathcal{N}=4$ splitting amplitudes and eikonal function [43]. Eq. (3.10) also determines the soft limit of two-loop splitting amplitudes beyond order $\epsilon^{0}$,

$$
\begin{aligned}
r_{S}^{(2)}(\epsilon)= & \frac{1}{8 \epsilon^{4}}+\frac{\zeta_{2}}{4 \epsilon^{2}}-\frac{11 \zeta_{3}}{24 \epsilon}+\frac{7 \zeta_{4}}{32}+\epsilon\left(-\frac{7 \zeta_{2} \zeta_{3}}{24}-\frac{247 \zeta_{5}}{40}\right) \\
& +\epsilon^{2}\left(-\frac{205 \zeta_{3}^{2}}{72}-\frac{3307 \zeta_{6}}{192}\right)+\mathcal{O}\left(\epsilon^{3}\right) .
\end{aligned}
$$

At three loops, the soft limit of splitting amplitudes at leading color is predicted to be [43]

$$
r_{S}^{(3)}(\epsilon)=-\frac{1}{3}\left(r_{S}^{(1)}(\epsilon)\right)^{3}+r_{S}^{(1)}(\epsilon) r_{S}^{(2)}(\epsilon)+f^{(3)}(\epsilon) r_{S}^{(1)}(3 \epsilon)+\mathcal{O}(\epsilon)
$$

where $f^{(3)}$ has been calculated through order $\epsilon^{2}[44]$,

$$
f^{(3)}(\epsilon)=\frac{11 \zeta_{4}}{2}+\left(5 \zeta_{2} \zeta_{3}+6 \zeta_{5}\right) \epsilon+a \epsilon^{2}+\mathcal{O}\left(\epsilon^{3}\right),
$$

with $a=85.263 \pm 0.004$. Using the above results, we obtain

$$
\begin{aligned}
r_{S}^{(3)}(\epsilon)= & -\frac{1}{48 \epsilon^{6}}-\frac{3 \zeta_{2}}{32 \epsilon^{4}}+\frac{\zeta_{3}}{12 \epsilon^{3}}-\frac{1487 \zeta_{4}}{2304 \epsilon^{2}}-\frac{13 \zeta_{2} \zeta_{3}}{144 \epsilon} \\
& +\frac{71 \zeta_{5}}{30 \epsilon}+\frac{11005 \zeta_{6}}{2048}+\frac{167 \zeta_{3}^{2}}{96}-\frac{a}{18}+\mathcal{O}(\epsilon) .
\end{aligned}
$$

\footnotetext{
${ }^{2}$ We are grateful to Lance Dixon for pointing us to the discussion in the rest of this section.
} 
For completeness, the $\mathcal{N}=4$ eikonal function at leading color is then given by

$$
S_{12, \mathcal{N}=4}^{(3)}(q)=8 S_{12}^{(0)}(q)\left(\frac{\alpha_{s}}{4 \pi}\right)^{3} S_{\epsilon}^{3} C_{A}^{3} r_{S}^{(3)}(\epsilon) .
$$

We note that eq. (3.14) is actually exact through order $\epsilon^{-1}$ for finite $N_{c}$. There are potential $\frac{1}{N_{c}}$ corrections, starting from order $\epsilon^{0}$. Unlike the one-loop and two-loop cases, these corrections would depend explicitly on the color representation of the hard partons, through the product of fourth order invariant tensor, $d_{R}^{i j k l} d_{A}^{i j k l}$. An explicit calculation of these corrections would be necessary in obtaining them.

Using the iterative predictions for the $\mathcal{N}=4$ splitting amplitudes [43] and the cusp anomalous dimension at leading color [66], the results above can further determine the leading-color $\mathcal{N}=4$ eikonal function at four loops through order $\epsilon^{-2}$.

\section{Conclusion}

In this paper we have computed the single soft-gluon current to two-loop order. we have compared our results with those in refs. $[18,19]$, and found full agreement to order $\epsilon^{0}$. The order $\epsilon$ and order $\epsilon^{2}$ terms presented in this paper are new. As a by-product, we have also given the soft-gluon current in $\mathcal{N}=4$ Super-Yang-Mills theory to order $\epsilon^{2}$, which in turn enables us to derive the splitting amplitudes in the soft limit, or the single soft-gluon current, at three loops and large $N_{c}$, using the results of refs. [43, 44]. We observe uniform transcendentality for the single soft-gluon current in $\mathcal{N}=4$ Super-Yang-Mills theory, and confirm that the leading transcendentality terms for the eikonal function are the same in QCD and $\mathcal{N}=4$ Super-Yang-Mills theory at two loops, up to order $\epsilon^{2}$.

The main purpose of the computation done in this paper is to provide the necessary ingredient for a calculation of Higgs production cross section at hadron collider at NNNLO. A lot of progress have been made recently in this direction [67-72]. A useful step towards the full NNNLO QCD corrections is the soft-virtual approximation at NNNLO. Using the results presented in this paper, the cross section for Higgs + one gluon emission can be computed by trivially integrating over the soft-gluon phase space. The cross section for Higgs +3 partons production in the soft limit has also been calculated recently in an impressive paper [70]. The only missing piece is the cross section for Higgs +2 partons production at one loop in the soft limit. It's reasonable to expect that the soft-virtual approximation for Higgs production at hadron collider at NNNLO will be available in the foreseeable future.

Besides Higgs physics, the soft gluon current at two loops is also useful in soft-collineareffective theory [50-53]. For example, the two-loop soft gluon current can be used to calculate soft function at NNNLO. Finally, we have only computed this soft gluon current with two energetic partons, or two Wilson lines. It's certainly interesting to extend our results to processes with an arbitrary number of Wilson lines. This will be relevant to jet physics at NNNLO. It will also be useful in understanding the structure of infrared divergences for multiple Wilson lines at three loops, see for example, ref. [73]. 


\section{Acknowledgments}

We are grateful to Lance Dixon for careful reading of the manuscript and invaluable suggestions. We also thank Michael Peskin for useful discussion. This research is supported by the US Department of Energy under contract DE-AC02-76SF00515. HXZ would like to thank Kavli Institute for Theoretical Physics at UC Santa Barbara and the Erwin Schrödinger Institute at University of Vienna for hospitality while part of this work was carried out. While this paper was being completed, we learned of independent work [74] by Claude Duhr and Thomas Gehrmann obtaining the soft-gluon current to all orders in $\epsilon$, which is in full agreement with the Laurent expansions of the soft-gluon current to order $\epsilon^{2}$ derived in this paper.

\section{A Evaluation of the master integral $I_{3}$ to order $\epsilon^{2}$}

In this appendix, we briefly explain the evaluation of the most difficult master integral, $I_{3}$. In fact, $I_{1}$ and $I_{2}$ can be obtained by deleting two propagators from $I_{3}$. We proceed by first performing the $k_{2}$ sub-loop integral by Feynman's trick,

$$
\begin{aligned}
I_{3}^{\prime} & =\int \frac{d^{D} k_{2}}{i \pi^{D / 2}} \frac{1}{\left[2\left(k_{2}+q\right) \cdot p_{2}\right]\left[k_{2}^{2}\right]\left[\left(k_{1}+k_{2}\right)^{2}\right]\left[\left(k_{2}+q\right)^{2}\right]} \\
& =(-1)^{D / 2} \Gamma\left(4-\frac{D}{2}\right) \int_{0}^{\infty} d y_{2} \int_{0}^{1} d x_{1} d x_{2} d x_{3} \frac{\delta\left(1-x_{1}-x_{2}-x_{3}\right)}{\Delta^{4-D / 2}},
\end{aligned}
$$

where

$$
\Delta=x_{1} x_{2}\left[k_{1}^{2}\right]+x_{1} x_{3}\left[\left(q+y_{2} p_{2}\right)^{2}\right]+x_{2} x_{3}\left[\left(k_{1}-q-y_{2} p_{2}\right)^{2}\right] .
$$

The resulting Feynman parameter integral over $d x_{i}$ can be factorized by introducing a two-fold Mellin-Barnes integral,

$$
\begin{aligned}
\frac{1}{\Delta^{4-D / 2}}= & \int_{-i \infty}^{+i \infty} \frac{d z_{1} d z_{2}}{(2 \pi i)^{2}} \Gamma\left(-z_{1}\right) \Gamma\left(-z_{2}\right) \frac{\Gamma\left(4-D / 2+z_{1}+z_{2}\right)}{\Gamma(4-D / 2)} \\
& \times\left(x_{1} x_{2}\left[k_{1}^{2}\right]\right)^{z_{1}}\left(x_{1} x_{3}\left[\left(q+y_{2} p_{2}\right)^{2}\right]\right)^{z_{2}}\left(x_{2} x_{3}\left[\left(k_{1}-q-y_{2} p_{2}\right)^{2}\right]\right)^{-z_{1}-z_{2}-4+D / 2}
\end{aligned}
$$

where the contour for $z_{i}$ separates the poles of $\Gamma\left(\cdots+z_{i}\right)$ from those of $\Gamma\left(\cdots-z_{i}\right)$. After this step, the Feynman parameter integral over $d x_{i}$ can be done in closed form in terms of $\Gamma$ functions. The remaining $k_{1}$ sub-loop integral has the form

$$
\int \frac{d^{D} k_{1}}{i \pi^{D / 2}} \frac{1}{\left[k_{1}^{2}\right]^{1-z_{1}}\left[\left(k_{1}-q-y_{2} p_{2}\right)^{2}\right]^{4-D / 2+z_{1}+z_{2}}\left[2 k_{1} \cdot p_{1}\right]\left[2\left(q-k_{1}\right) \cdot p_{2}\right]},
$$

which can be straightforwardly done. We then arrive at a two-fold Mellins-Barnes integral representation for $I_{3}$,

$$
\begin{aligned}
I_{3}= & \frac{1}{8\left(16 \pi^{2}\right)^{2}} S_{\epsilon}^{2} e^{2 \epsilon \gamma_{E}} \Gamma(5-D) \\
& \times \int_{-i \infty}^{+i \infty} \frac{d z_{1} d z_{2}}{(2 \pi i)^{2}} \Gamma\left(-z_{1}\right) \Gamma\left(-z_{2}\right) \Gamma\left(z_{2}+1\right) \Gamma\left(\frac{D}{2}-z_{1}-2\right) \Gamma\left(\frac{D}{2}+z_{1}-2\right) \\
& \times \frac{\Gamma\left(\frac{D}{2}-z_{2}-3\right) \Gamma\left(-D+z_{2}+6\right) \Gamma\left(1+z_{1}+z_{2}\right) \Gamma\left(D-z_{1}-z_{2}-5\right)}{\Gamma\left(1-z_{1}\right) \Gamma\left(2+z_{2}\right) \Gamma\left(\frac{3 D}{2}-z_{2}-7\right)} .
\end{aligned}
$$


We were not able to find an all order in $\epsilon$ solution of this integral. Instead, we calculate the Laurent expansion of the Mellin-Barnes integral to order $\epsilon^{2}$, which is relevant for NNNLO phenomenology. To that end, we make use of the MATHEMATICA pachages MB [63] and BARNESROUTINES of D. Kosower to resolve the singularity, to expand the integrand in $\epsilon$, and to apply the Barnes lemma in an automatic way, which results in a series of one-fold and two-fold Mellin-Barnes integrals. The one-fold integral can easily be done numerically using MATHEMATICA's NIntegrate routine, and the results can be converted into Riemann zeta values using the PSLQ algorithm $[75,76]$. The only remaining two-fold Mellin-Barnes integral is

$$
\begin{array}{r}
\int_{-i \infty}^{+i \infty} \frac{d z_{1} d z_{2}}{(2 \pi i)^{2}} \frac{\Gamma\left(-z_{1}\right)^{2} \Gamma\left(z_{1}\right) \Gamma\left(-z_{2}\right) \Gamma\left(1+z_{2}\right) \Gamma\left(-1-z_{1}-z_{2}\right) \Gamma\left(1+z_{1}+z_{2}\right)}{\Gamma\left(1-z_{1}\right)} \\
\times\left(\psi\left(-1-z_{2}\right)+\psi\left(2+z_{2}\right)\right)\left(2 \psi\left(-1-z_{1}-z_{2}\right)+\psi\left(-z_{1}\right)+\psi\left(z_{1}\right)\right)
\end{array}
$$

where $\psi(x)$ is the logarithmic derivative of $\Gamma$ function, and the integration contours are straight vertical lines defined by

$$
\operatorname{Re}\left(z_{1}\right)=-\frac{1091}{1641}, \quad \operatorname{Re}\left(z_{2}\right)=-\frac{554}{1671} .
$$

The integral can be performed by closing the contour to the left or right, and summing up the residues at the poles. The results are double sums of the form

$$
\sum_{m, n=1}^{\infty} \frac{S_{\vec{i}_{1}}(m)}{m^{j_{1}}} \frac{S_{\vec{i}_{2}}(n)}{n^{j_{2}}} \frac{S_{\vec{i}_{3}}(m+n)}{(m+n)^{j_{3}}},
$$

where $S_{\vec{i}}(k)$ are nested harmonic sums defined in ref. [77]. The summation can be conveniently done using XSummer [78]. The final result for this master integral is checked numerically using the package FIESTA [79] and the author's personal tool, based on the method of sector decomposition [80].

Open Access. This article is distributed under the terms of the Creative Commons Attribution License which permits any use, distribution and reproduction in any medium, provided the original author(s) and source are credited.

\section{References}

[1] G. Altarelli and G. Parisi, Asymptotic freedom in parton language, Nucl. Phys. B 126 (1977) 298 [inSPIRE].

[2] A. Bassetto, M. Ciafaloni and G. Marchesini, Jet structure and infrared sensitive quantities in perturbative QCD, Phys. Rept. 100 (1983) 201 [INSPIRE].

[3] F.A. Berends and W. Giele, Multiple soft gluon radiation in parton processes, Nucl. Phys. B 313 (1989) 595 [INSPIRE].

[4] Y.L. Dokshitzer, V.A. Khoze, A.H. Mueller and S. Troian, Basics of perturbative QCD, Editions Frontières, Gif-sur-Yvette France (1991). 
[5] R.K. Ellis, W.J. Stirling and B. Webber, $Q C D$ and collider physics, Cambridge University Press, Cambridge U.K. (1996).

[6] J.M. Campbell and E.N. Glover, Double unresolved approximations to multiparton scattering amplitudes, Nucl. Phys. B 527 (1998) 264 [hep-ph/9710255] [InSPIRE].

[7] S. Catani and M. Grazzini, Infrared factorization of tree level QCD amplitudes at the next-to-next-to-leading order and beyond, Nucl. Phys. B 570 (2000) 287 [hep-ph/9908523] [INSPIRE].

[8] Z. Bern and G. Chalmers, Factorization in one loop gauge theory, Nucl. Phys. B 447 (1995) 465 [hep-ph/9503236] [INSPIRE].

[9] D.A. Kosower, All order collinear behavior in gauge theories, Nucl. Phys. B 552 (1999) 319 [hep-ph/9901201] [INSPIRE].

[10] S. Catani and M. Grazzini, Collinear factorization and splitting functions for next-to-next-to-leading order QCD calculations, Phys. Lett. B 446 (1999) 143 [hep-ph/9810389] [INSPIRE].

[11] V. Del Duca, A. Frizzo and F. Maltoni, Factorization of tree QCD amplitudes in the high-energy limit and in the collinear limit, Nucl. Phys. B 568 (2000) 211 [hep-ph/9909464] [INSPIRE].

[12] Z. Bern, V. Del Duca and C.R. Schmidt, The infrared behavior of one loop gluon amplitudes at next-to-next-to-leading order, Phys. Lett. B 445 (1998) 168 [hep-ph/9810409] [INSPIRE].

[13] S. Catani, The singular behavior of QCD amplitudes at two loop order, Phys. Lett. B 427 (1998) 161 [hep-ph/9802439] [INSPIRE].

[14] Z. Bern, V. Del Duca, W.B. Kilgore and C.R. Schmidt, The infrared behavior of one loop QCD amplitudes at next-to-next-to leading order, Phys. Rev. D 60 (1999) 116001 [hep-ph/9903516] [INSPIRE].

[15] S. Catani and M. Grazzini, The soft gluon current at one loop order, Nucl. Phys. B 591 (2000) 435 [hep-ph/0007142] [INSPIRE].

[16] D.A. Kosower, All orders singular emission in gauge theories, Phys. Rev. Lett. 91 (2003) 061602 [hep-ph/0301069] [INSPIRE].

[17] S. Catani, D. de Florian and G. Rodrigo, The triple collinear limit of one loop QCD amplitudes, Phys. Lett. B 586 (2004) 323 [hep-ph/0312067] [INSPIRE].

[18] Z. Bern, L.J. Dixon and D.A. Kosower, Two-loop $g \rightarrow$ gg splitting amplitudes in QCD, JHEP 08 (2004) 012 [hep-ph/0404293] [INSPIRE].

[19] S. Badger and E.N. Glover, Two loop splitting functions in QCD, JHEP 07 (2004) 040 [hep-ph/0405236] [INSPIRE].

[20] A. Gehrmann-De Ridder, T. Gehrmann, E. Glover and G. Heinrich, Infrared structure of $e^{+} e^{-} \rightarrow 3$ jets at NNLO, JHEP 11 (2007) 058 [arXiv:0710.0346] [INSPIRE].

[21] I. Bierenbaum, M. Czakon and A. Mitov, The singular behavior of one-loop massive QCD amplitudes with one external soft gluon, Nucl. Phys. B 856 (2012) 228 [arXiv:1107.4384] [INSPIRE].

[22] S. Catani, D. de Florian and G. Rodrigo, Space-like (versus time-like) collinear limits in QCD: is factorization violated?, JHEP 07 (2012) 026 [arXiv:1112.4405] [INSPIRE]. 
[23] J. Currie, E.W.N. Glover and S. Wells, Infrared structure at NNLO using antenna subtraction, JHEP 04 (2013) 066 [arXiv: 1301.4693] [INSPIRE].

[24] I. Feige and M.D. Schwartz, An on-shell approach to factorization, Phys. Rev. D 88 (2013) 065021 [arXiv: 1306.6341] [INSPIRE].

[25] A. Gehrmann-De Ridder, T. Gehrmann and E.N. Glover, Antenna subtraction at NNLO, JHEP 09 (2005) 056 [hep-ph/0505111] [INSPIRE].

[26] G. Somogyi, Z. Trócsányi and V. Del Duca, Matching of singly- and doubly-unresolved limits of tree-level QCD squared matrix elements, JHEP 06 (2005) 024 [hep-ph/0502226] [INSPIRE].

[27] M. Czakon, A novel subtraction scheme for double-real radiation at NNLO, Phys. Lett. B 693 (2010) 259 [arXiv: 1005.0274] [INSPIRE].

[28] P. Bärnreuther, M. Czakon and A. Mitov, Percent level precision physics at the Tevatron: first genuine NNLO QCD corrections to $q \bar{q} \rightarrow t \bar{t}+X$, Phys. Rev. Lett. 109 (2012) 132001 [arXiv: 1204.5201] [INSPIRE].

[29] M. Czakon, P. Fiedler and A. Mitov, The total top quark pair production cross-section at hadron colliders through $O\left(\right.$ alpha $\left._{S}^{4}\right)$, Phys. Rev. Lett. 110 (2013) 252004 [arXiv:1303.6254] [INSPIRE].

[30] A. Gehrmann-De Ridder, T. Gehrmann, E. Glover and J. Pires, Second order QCD corrections to jet production at hadron colliders: the all-gluon contribution, Phys. Rev. Lett. 110 (2013) 162003 [arXiv:1301.7310] [INSPIRE].

[31] R. Boughezal, F. Caola, K. Melnikov, F. Petriello and M. Schulze, Higgs boson production in association with a jet at next-to-next-to-leading order in perturbative $Q C D$, JHEP 06 (2013) 072 [arXiv: 1302.6216] [INSPIRE].

[32] ATLAS collaboration, Observation of a new particle in the search for the standard model Higgs boson with the ATLAS detector at the LHC, Phys. Lett. B 716 (2012) 1 [arXiv: 1207.7214] [INSPIRE].

[33] CMS collaboration, Observation of a new boson at a mass of $125 \mathrm{GeV}$ with the CMS experiment at the LHC, Phys. Lett. B 716 (2012) 30 [arXiv:1207.7235] [INSPIRE].

[34] R.V. Harlander and W.B. Kilgore, Next-to-next-to-leading order Higgs production at hadron colliders, Phys. Rev. Lett. 88 (2002) 201801 [hep-ph/0201206] [INSPIRE].

[35] C. Anastasiou and K. Melnikov, Higgs boson production at hadron colliders in NNLO QCD, Nucl. Phys. B 646 (2002) 220 [hep-ph/0207004] [INSPIRE].

[36] V. Ravindran, J. Smith and W.L. van Neerven, NNLO corrections to the total cross-section for Higgs boson production in hadron hadron collisions, Nucl. Phys. B 665 (2003) 325 [hep-ph/0302135] [INSPIRE].

[37] S. Catani and M. Grazzini, An NNLO subtraction formalism in hadron collisions and its application to Higgs boson production at the LHC, Phys. Rev. Lett. 98 (2007) 222002 [hep-ph/0703012] [INSPIRE].

[38] LHC Higgs Cross Section Working Group collaboration, S. Dittmaier et al., Handbook of LHC Higgs cross sections: 1. Inclusive observables, arXiv:1101.0593 [INSPIRE].

[39] R. Boughezal, K. Melnikov and F. Petriello, A subtraction scheme for NNLO computations, Phys. Rev. D 85 (2012) 034025 [arXiv:1111.7041] [INSPIRE]. 
[40] L. Garland, T. Gehrmann, E.N. Glover, A. Koukoutsakis and E. Remiddi, The two loop QCD matrix element for $e^{+} e^{-} \rightarrow 3$ jets, Nucl. Phys. B 627 (2002) 107 [hep-ph/0112081] [INSPIRE].

[41] L. Garland, T. Gehrmann, E.N. Glover, A. Koukoutsakis and E. Remiddi, Two loop QCD helicity amplitudes for $e^{+} e^{-} \rightarrow 3$ jets, Nucl. Phys. B 642 (2002) 227 [hep-ph/0206067] [INSPIRE].

[42] T. Gehrmann, M. Jaquier, E. Glover and A. Koukoutsakis, Two-loop QCD corrections to the helicity amplitudes for $H \rightarrow 3$ partons, JHEP 02 (2012) 056 [arXiv:1112.3554] [INSPIRE].

[43] Z. Bern, L.J. Dixon and V.A. Smirnov, Iteration of planar amplitudes in maximally supersymmetric Yang-Mills theory at three loops and beyond, Phys. Rev. D 72 (2005) 085001 [hep-th/0505205] [INSPIRE].

[44] M. Spradlin, A. Volovich and C. Wen, Three-loop leading singularities and BDS ansatz for five particles, Phys. Rev. D 78 (2008) 085025 [arXiv:0808.1054] [InSPIRE].

[45] J. Gatheral, Exponentiation of eikonal cross-sections in nonabelian gauge theories, Phys. Lett. B 133 (1983) 90 [INSPIRE].

[46] J. Frenkel and J. Taylor, Nonabelian eikonal exponentiation, Nucl. Phys. B 246 (1984) 231 [INSPIRE].

[47] S. Catani, D. de Florian and M. Grazzini, Higgs production in hadron collisions: soft and virtual QCD corrections at NNLO, JHEP 05 (2001) 025 [hep-ph/0102227] [INSPIRE].

[48] R.V. Harlander and W.B. Kilgore, Soft and virtual corrections to proton proton $\rightarrow H+x$ at NNLO, Phys. Rev. D 64 (2001) 013015 [hep-ph/0102241] [INSPIRE].

[49] D. de Florian and J. Mazzitelli, A next-to-next-to-leading order calculation of soft-virtual cross sections, JHEP 12 (2012) 088 [arXiv:1209.0673] [INSPIRE].

[50] C.W. Bauer, S. Fleming and M.E. Luke, Summing Sudakov logarithms in $B \rightarrow X(s \gamma)$ in effective field theory, Phys. Rev. D 63 (2000) 014006 [hep-ph/0005275] [INSPIRE].

[51] C.W. Bauer, S. Fleming, D. Pirjol and I.W. Stewart, An effective field theory for collinear and soft gluons: heavy to light decays, Phys. Rev. D 63 (2001) 114020 [hep-ph/0011336] [INSPIRE].

[52] C.W. Bauer, D. Pirjol and I.W. Stewart, Soft collinear factorization in effective field theory, Phys. Rev. D 65 (2002) 054022 [hep-ph/0109045] [INSPIRE].

[53] M. Beneke, A. Chapovsky, M. Diehl and T. Feldmann, Soft collinear effective theory and heavy to light currents beyond leading power, Nucl. Phys. B 643 (2002) 431 [hep-ph/0206152] [INSPIRE].

[54] P. Nogueira, Automatic Feynman graph generation, J. Comput. Phys. 105 (1993) 279.

[55] K. Chetyrkin and F. Tkachov, Integration by parts: the algorithm to calculate $\beta$-functions in 4 loops, Nucl. Phys. B 192 (1981) 159 [INSPIRE].

[56] F. Tkachov, A theorem on analytical calculability of four loop renormalization group functions, Phys. Lett. B 100 (1981) 65 [INSPIRE].

[57] A. Smirnov, Algorithm FIRE - Feynman Integral REduction, JHEP 10 (2008) 107 [arXiv: 0807.3243] [INSPIRE].

[58] S. Laporta, High precision calculation of multiloop Feynman integrals by difference equations, Int. J. Mod. Phys. A 15 (2000) 5087 [hep-ph/0102033] [inSPIRE]. 
[59] R. Lee, Presenting LiteRed: a tool for the Loop InTEgrals REDuction, arXiv:1212.2685 [INSPIRE].

[60] Z. Bern and D.A. Kosower, The computation of loop amplitudes in gauge theories, Nucl. Phys. B 379 (1992) 451 [inSPIRE].

[61] Z. Bern, A. De Freitas, L.J. Dixon and H. Wong, Supersymmetric regularization, two loop QCD amplitudes and coupling shifts, Phys. Rev. D 66 (2002) 085002 [hep-ph/0202271] [INSPIRE].

[62] T. Gehrmann and E. Remiddi, Two loop master integrals for $\gamma^{*} \rightarrow 3$ jets: the planar topologies, Nucl. Phys. B 601 (2001) 248 [hep-ph/0008287] [INSPIRE].

[63] M. Czakon, Automatized analytic continuation of Mellin-Barnes integrals, Comput. Phys. Commun. 175 (2006) 559 [hep-ph/0511200] [INSPIRE].

[64] A. Kotikov, L. Lipatov, A. Onishchenko and V. Velizhanin, Three loop universal anomalous dimension of the Wilson operators in $N=4$ SUSY Yang-Mills model, Phys. Lett. B 595 (2004) 521 [Erratum ibid. B 632 (2006) 754-756] [hep-th/0404092] [INSPIRE].

[65] C. Anastasiou, Z. Bern, L.J. Dixon and D. Kosower, Planar amplitudes in maximally supersymmetric Yang-Mills theory, Phys. Rev. Lett. 91 (2003) 251602 [hep-th/0309040] [INSPIRE].

[66] Z. Bern, M. Czakon, L.J. Dixon, D.A. Kosower and V.A. Smirnov, The four-loop planar amplitude and cusp anomalous dimension in maximally supersymmetric Yang-Mills theory, Phys. Rev. D 75 (2007) 085010 [hep-th/0610248] [INSPIRE].

[67] A. Pak, M. Rogal and M. Steinhauser, Production of scalar and pseudo-scalar Higgs bosons to next-to-next-to-leading order at hadron colliders, JHEP 09 (2011) 088 [arXiv:1107.3391] [INSPIRE].

[68] C. Anastasiou, S. Buehler, C. Duhr and F. Herzog, NNLO phase space master integrals for two-to-one inclusive cross sections in dimensional regularization, JHEP 11 (2012) 062 [arXiv:1208.3130] [INSPIRE].

[69] M. Höschele, J. Hoff, A. Pak, M. Steinhauser and T. Ueda, Higgs boson production at the LHC: NNLO partonic cross sections through order $\epsilon$ and convolutions with splitting functions to $N^{3} L O$, Phys. Lett. B 721 (2013) 244 [arXiv:1211.6559] [INSPIRE].

[70] C. Anastasiou, C. Duhr, F. Dulat and B. Mistlberger, Soft triple-real radiation for Higgs production at N3LO, JHEP 07 (2013) 003 [arXiv:1302.4379] [INSPIRE].

[71] R.D. Ball, M. Bonvini, S. Forte, S. Marzani and G. Ridolfi, Higgs production in gluon fusion beyond NNLO, Nucl. Phys. B 874 (2013) 746 [arXiv:1303.3590] [inSPIRE].

[72] S. Buehler and A. Lazopoulos, Scale dependence and collinear subtraction terms for Higgs production in gluon fusion at N3LO, JHEP 10 (2013) 096 [arXiv:1306.2223] [INSPIRE].

[73] E. Gardi, J.M. Smillie and C.D. White, The non-abelian exponentiation theorem for multiple Wilson lines, JHEP 06 (2013) 088 [arXiv: 1304.7040] [INSPIRE].

[74] C. Duhr and T. Gehrmann, The two-loop soft current in dimensional regularization, arXiv: 1309.4393 [INSPIRE].

[75] H.R.P. Ferguson and D.H. Bailey, A polynomial time, numerically stble integer relationalgorithm, RNR-91-032 (1991). 
[76] H.R.P. Ferguson, D.H. Bailey and S. Arno, Analysis of PSLQ, an integer relation finding algorithm, Math. Comput. 68 (1999) 351.

[77] J. Vermaseren, Harmonic sums, Mellin transforms and integrals, Int. J. Mod. Phys. A 14 (1999) 2037 [hep-ph/9806280] [inSPIRE].

[78] S. Moch and P. Uwer, XSummer: transcendental functions and symbolic summation in form, Comput. Phys. Commun. 174 (2006) 759 [math-ph/0508008] [INSPIRE].

[79] A. Smirnov, V. Smirnov and M. Tentyukov, FIESTA 2: parallelizeable multiloop numerical calculations, Comput. Phys. Commun. 182 (2011) 790 [arXiv:0912.0158] [INSPIRE].

[80] T. Binoth and G. Heinrich, An automatized algorithm to compute infrared divergent multiloop integrals, Nucl. Phys. B 585 (2000) 741 [hep-ph/0004013] [INSPIRE]. 